\title{
Influence of Substrate Material on Radiation Characteristics of THz Photoconductive Emitters
}

\author{
Jens Klier, ${ }^{1}$ Garik Torosyan, ${ }^{1}$ Nina Susan Schreiner, ${ }^{1}$ Daniel Molter, ${ }^{1}$ \\ Frank Ellrich, ${ }^{1}$ Wissem Zouaghi, ${ }^{2}$ Emilien Peytavit, ${ }^{3}$ Jean-François Lampin, ${ }^{3}$ \\ René Beigang, ${ }^{4}$ Joachim Jonuscheit, ${ }^{1}$ and Georg von Freymann ${ }^{1,4}$ \\ ${ }^{1}$ Fraunhofer Institute for Physical Measurement Techniques IPM, Fraunhofer-Platz 1, 67663 Kaiserslautern, Germany
${ }^{2}$ Physikalisches Institut, Goethe Universität, 60438 Frankfurt am Main, Germany
${ }^{3}$ Institut d'Electronique, Microélectronique et de Nanotechnologie (IEMN), Villeneuve-d'Ascq Cedex, 59652 Lille, France
${ }^{4}$ Department of Physics and Research Center OPTIMAS, University of Kaiserslautern, 67663 Kaiserslautern, Germany
}

Correspondence should be addressed to Garik Torosyan; garik.torosyan@ipm.fraunhofer.de

Received 20 April 2015; Revised 2 August 2015; Accepted 24 August 2015

Academic Editor: Francisco Falcone

Copyright (C) 2015 Jens Klier et al. This is an open access article distributed under the Creative Commons Attribution License, which permits unrestricted use, distribution, and reproduction in any medium, provided the original work is properly cited.

\begin{abstract}
We present in this paper spectral and spatial characteristics of terahertz emission from standard dipole antenna structures used as emitters depending on the substrate material. All antenna structures were lithographically fabricated on low-temperature (LT) grown, few-micrometers-thick gallium arsenide (GaAs) layers. To investigate the effect of the substrate material on the radiation pattern of terahertz beams, either semi-insulating gallium arsenide or high-resistivity silicon substrate wafers have been used. As detector a standard $40 \mu \mathrm{m}$ long dipole antenna on a semi-insulating GaAs substrate with a low-temperature grown gallium arsenide layer on it has been employed; this configuration allows for broadband detection and is still efficient enough for the characterization purpose. Strong dependence of the radiation pattern on the substrate used for the terahertz source is demonstrated. The measured patterns and differences between the two cases of substrates are well explained by means of classical diffraction.
\end{abstract}

\section{Introduction}

In the electromagnetic spectrum, the region between roughly $10^{11} \mathrm{~Hz}$ and $10^{13} \mathrm{~Hz}$ is referred to as the terahertz (THz) region: $1 \mathrm{THz}=10^{12} \mathrm{~Hz}$. Due to the low photon energy, such invisible radiation is harmless to the human body, to biomolecules, to food, and to pharmaceutics $[1,2]$. Corresponding techniques are being developed and used in applications such as civil security $[3,4]$ or quality and process control $[5,6]$. With the development of femtosecond lasers in the 1980s, THz research has become more intense thanks to the development of a new emission and detection methodthe photoconductive switch [7-11]. The operation principle of the photoconductive switch used as an antenna for $\mathrm{THz}$ radiation is the following: Two symmetric strip lines are arranged on a semiconducting substrate with the symmetrically positioned dipole having a $5 \mu \mathrm{m}$ long gap in the center (see Figure 1). In the case of the emitter antenna these are supplied with a DC bias voltage source $\left(U_{b}\right)$, which leads to an electric bias field between them. Irradiating the antenna with a femtosecond ( $f s$ ) laser pulse focused on the dipole gap, the substrate gets temporarily conductive. The generated short lifetime carriers will be accelerated through the bias voltage giving rise to a current transient of subpicosecond duration flowing through the antenna. The shape and the duration of this transient depend on the laser pulse itself and the semiconductor's physical properties, that is, the lifetime of the generated free charge carriers. According to Maxwell's theory the current transient induces a pulsed electromagnetic wave of corresponding duration to be emitted into the free space. In this particular case it is the $\mathrm{THz}$ pulse through which all the spectroscopic measurements are performed. In a typical case the lifetime lies below $500 \mathrm{fs}$; hence, the antenna is inactive until the next laser pulse arrives after typical $10 \mathrm{~ns}$ time, and everything is replayed in the same way with every next laser pulse. A similar but not biased antenna may operate 


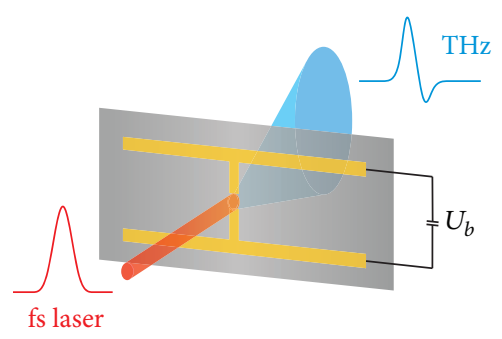

FIGURE 1: Operation principle of photoconductive antennas. The laser beam is focused on the dipole gap, the dipole gap, generating electron-hole pairs, and the transient current conditioned by the applied voltage $U_{b}$ emits an electromagnetic pulse of subpicosecond duration-the $\mathrm{THz}$ pulse-into the free space. The emitted wavelengths are much longer compared to the size of their source, the dipole; hence, they diffract strongly in the form of a wide cone filling out almost the whole solid angle behind the antenna (dark grey area marked $\mathrm{THz}$ ).

inversely as a detector, to detect and measure freely travelling $\mathrm{THz}$ waves. Instead of applying a DC voltage, the antenna in this case is connected to the input of a nanoampere meter.

The latter is used for measuring the short electric current transient induced by the $\mathrm{THz}$ pulse that covers the antenna dipole if it is correspondingly aligned. Due to the very weak signals the response of the antenna to the $\mathrm{THz}$ electric field is linear and the temporal form of the measured current amplitude repeats that of the electric field of the $\mathrm{THz}$ pulse. To make the operation of the antennas efficient a silicon lens is glued or pressed on the back side of the antenna substrate. For our applications we have been using hyperhemispherical lenses made of high-resistivity silicon (HR-Si) with $10 \mathrm{~mm}$ diameter and $6.15 \mathrm{~mm}$ height. By varying the geometrical [12] and material parameters of the antenna structures, the $\mathrm{THz}$ field may be optimized for the respective application such as spectroscopy or imaging. The influence of substrate material of the antenna on the radiation pattern of the $\mathrm{THz}$ radiation in the far field is the subject of this paper. The measured electric signals (time domain) are further amplified and by means of Fourier transformation analyzed spectroscopically (frequency domain) afterwards. The $\mathrm{THz}$ pulse on its way from the emitter to the detector acquires specific information of the environment or an object through which it has passed and reveals its spectral characteristics after the Fourier transform. This technique of doing measurements in time domain and analyzing results in frequency domain is called $\mathrm{THz}$ time domain spectroscopy (THz-TDS) [13, 14]. Moving the object across the $\mathrm{THz}$ beam and performing TDS measurements for each position make it possible to get $\mathrm{THz}$ images of the object under investigation. For that purpose focused $\mathrm{THz}$ beams are mostly used and hence it is important to have exact information about the spectral and spatial quality of the generated $\mathrm{THz}$ beam.

\section{Setup}

The schematic of the used THz-TDS experimental setup is depicted in Figure 2. The optical pulse from the femtosecond

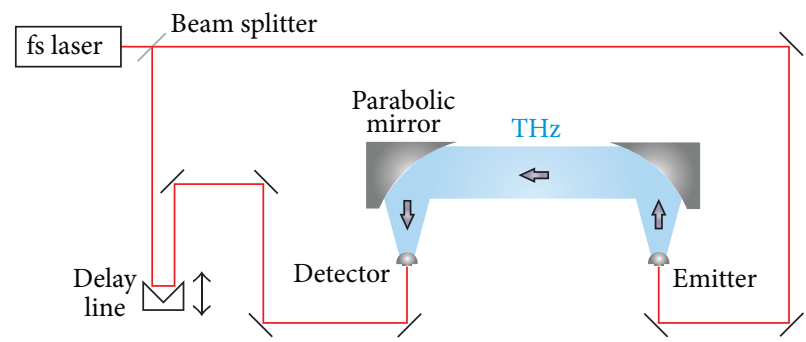

FIgURE 2: Schematic of a standard THz-TDS experimental setup. The optical pulse is split into two halves. The one through the beam splitter reaches the emitter antenna after a fixed flight time and gives rise to the $\mathrm{THz}$ pulse to be emitted into the free space and travel through $\mathrm{THz}$ optics to the detector. The reflected part of the optical pulse is delayed by means of the delay line before reaching the detector antenna against the arrival time of the $\mathrm{THz}$ pulse. Depending on the delay line position the optical pulse "opens" the antenna to detect different elementary sections along the electric field amplitude envelope of $\mathrm{THz}$ pulse.

laser (central wavelength $800 \mathrm{~nm}, 120 \mathrm{fs}$ pulse duration, and $80 \mathrm{MHz}$ repetition rate [15]) is split into two beams with a beam splitter. One arm, led by the transmitted part, travels to the emitter antenna and reaches it after a fixed time and "opens" it giving rise to the $\mathrm{THz}$ pulse to be emitted into the free space from the back side of the antenna. The $\mathrm{THz}$ pulse travels through reflective $\mathrm{THz}$ optics to the detector and completes the emitter arm. The reflected beam from the beam splitter enters the second arm. It can be intentionally delayed by means of the delay line before reaching the detector antenna to match the arrival time of the $\mathrm{THz}$ pulse to open the detector. As long as the detector is open, the incoming $\mathrm{THz}$ pulse is able to induce a current transient through the antenna structure proportional to its immediate amplitude. Depending on the delay line position the optical pulse "opens" the antenna to detect different elementary sections along the electric field amplitude envelope of the $\mathrm{THz}$ pulse. Since the generated photocurrents are very low, lock-in technique is used to amplify the signal for further processing.

As THz emitter a $20 \mu \mathrm{m}$ dipole antenna consisting of lowtemperature grown gallium arsenide (LT-GaAs) on a semiinsulating gallium arsenide (SI-GaAs) substrate was used. In a second experiment the emitter was exchanged by LTGaAs on a high-resistivity (HR) silicon substrate [14]. The approximately $2 \mu \mathrm{m}$ thin LT-GaAs layer was bonded onto the HR-Si substrate by natural intermolecular surface forces (Van der Waals bonding). This LT-GaAs layer is just large enough to process the dipole antenna on it. At the edge the wire contacts are bonded to wires on the Si substrate so one can connect it to the circuit board (see Figure 3).

It is possible with this approach to use the LT-GaAs wafer more efficiently and to process hundreds of $\mathrm{THz}$ antennas from one wafer. To be sure that the emitted $\mathrm{THz}$ radiation shows symmetric field distribution the Si lens needs to be aligned precisely. Thus, in addition to the dipole antenna 4 alignment marks were processed on the chip. With the help of those marks it is possible to determine the exact position 


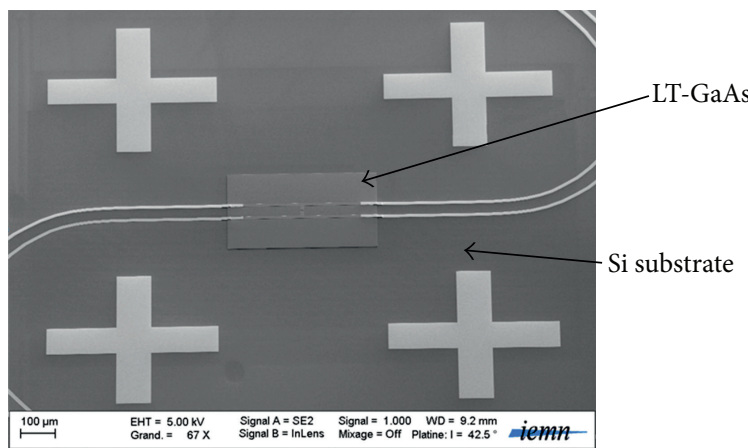

FIGURE 3: The rectangle in the center is a thin LT-GaAs layer on the HR-Si substrate. On the layer the dipole antenna and in each corner the alignment marks are processed. Wires on the substrate are connected to the dipole on the layer.

of the dipole antenna with respect to the optical axis of the Si lens by using a high resolution camera. The lens is mounted on the back side of the chip, and the edge of this lens can be captured from the camera too. Thus, the center position of the lens can be determined. With two stepper motors in $x$ - and $y$-direction it is possible to align the center position of the $\mathrm{Si}$ lens on the dipole antenna with an accuracy of $2 \mu \mathrm{m}$. In the following step the Si lens is glued with a UV adhesive onto the chip.

To scan a range of $50 \mathrm{~mm}$ in $x$-, $y$-, and $z$-direction, a fiber-coupled photoconductive switch (PCS) as THz detector based on a $40 \mu \mathrm{m}$ dipole antenna was used to avoid realigning the optical part after every movement. In Figure 4, a schematic drawing of our setup and one measured beam profile in accordance with that scheme are shown.

Furthermore, it was possible to change the distance in $z$ direction between the $\mathrm{THz}$ far-field radiation pattern from photoconductive emitters on different substrates and the $\mathrm{THz}$ detector in a range from $30 \mathrm{~mm}$ to $80 \mathrm{~mm}$. At each detector position a $\mathrm{THz}$-TDS scan with a spectral bandwidth of approximately $2 \mathrm{THz}$ was performed. The emitted average power for both antenna geometries was in the order of a few $\mu \mathrm{W}$.

\section{Results}

In previous work far-field measurements using a sapphire substrate $(n=3.40)$ and a Si lens have shown a frequency dependent $\mathrm{THz}$ field distribution [16]. Based on these results we have first performed measurements with a GaAs substrate on a Si lens. In our setup the thickness of this substrate was $400 \mu \mathrm{m}$. The refractive index step of the different materials (0.19 in this case) leads to total internal reflection and, as a consequence, to a limiting stop aperture of approximately $2.2 \mathrm{~mm}$ as shown in Figure 5(a). Second, we compare these results with LT-GaAs on a Si substrate, again in combination with a Si lens. The advantage is that now there is no stop aperture at the interface because the substrate and the lens are of the same material. In this case the limiting aperture is given by the dimensions of the lens itself (see Figure 5(b)).
The thickness of the LT-GaAs layer $(2 \mu \mathrm{m})$ is small compared to the emitted wavelength of the $\mathrm{THz}$ radiation; hence, the interfaces between the small LT-GaAs emitter and the surrounding Si can be neglected, acting as a bulk Si lens with integrated emitter.

This behavior (schematically shown in Figure 5) and the influence of the resulting diffraction at the stop aperture on the radiation pattern were experimentally verified for a $\mathrm{THz}$ beam emitted by the emitter based on GaAs substrate. The measured waveforms for both configurations are shown in Figure 6. No significant change is visible; the additional echoes are due to nonperfect attachment of the Si substrate to the Si lens. The signal strength depends strongly on the alignment of the Si lens and on the quality of the surface between LT-GaAs layer and Si substrate. Any scratch or dust reduces significantly the signal strength. The spectrum shows some oscillations due to the echoes in the waveforms. The signal-to-noise ratios (SNR) are comparable.

The measured distributions of electric field strength for various frequency components in the $X Z$-plane are shown in Figure 7. As one can see, for lower frequencies around $200 \mathrm{GHz}$ one has almost a diffraction-free picture. At higher frequencies around $550 \mathrm{GHz}$ an obvious interference minimum between 50 and $80 \mathrm{~mm}$ and one maximum ring are observed. At $900 \mathrm{GHz}$ the depth of the central minimum is getting smaller, while the next diffraction order in the form of the second ring can be detected. At $1.2 \mathrm{THz}$ the picture is getting even more complicated: Higher orders of diffraction appear.

The measured and numerically calculated frequencyresolved radiation patterns for a SI-GaAs substrate taken from the measurements at the distance of $65 \mathrm{~mm}$ (marked by the blue lines in Figure 7) from the emitter are shown in Figure 8. As seen experimentally from the pattern we have observed up to 4 diffraction fringes in the frequency range from $0 \mathrm{THz}$ up to $1.8 \mathrm{THz}$. This intensity distribution is a result of strong diffraction caused by the stop aperture which is formed due to the step between the refractive indices of the antenna substrate and the Si lens. For the calculation we numerically solve the Kirchhoff integral, neglecting absorption in $\mathrm{Si}$ and in GaAs, as they are negligible in the spectral region investigated. The antenna is represented by a dipole. The numerical calculation shows a qualitative similar behavior. However, the correspondence with the experimental data could be improved by better determining the material's parameter; here, we used the refractive indices stated in the paper.

In contrast to the emitter with SI-GaAs the radiation patterns from the emitter with HR-Si substrate are more collimated and much simpler in cross section. A corresponding beam profile is shown in Figure 9. As one can see there are no strong interference effects or higher-order diffraction effects in the same frequency range anymore. As expected, the beam profile narrows down for increasing frequencies. Much more homogeneous spatial distributions are advantageous for spectrally resolved imaging application, as there are no longer any dark spots found on the sample surface, which might lead to frequency depending imaging artifacts. 


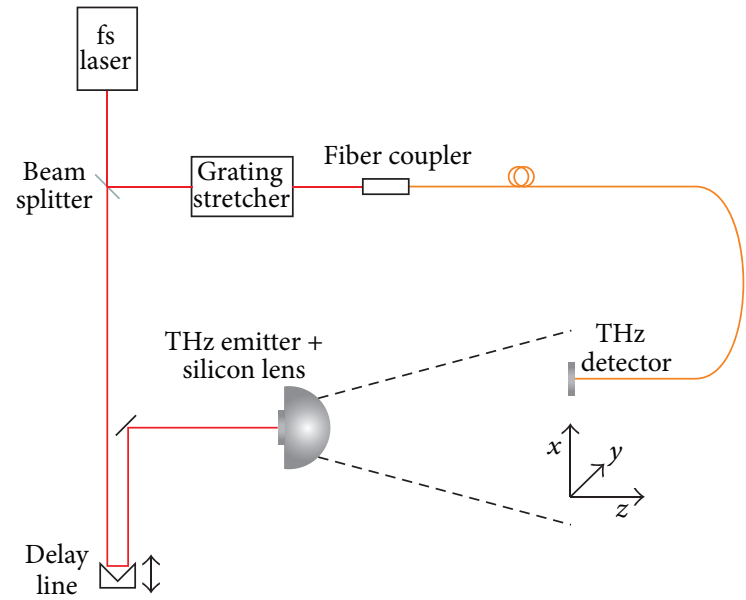

(a)

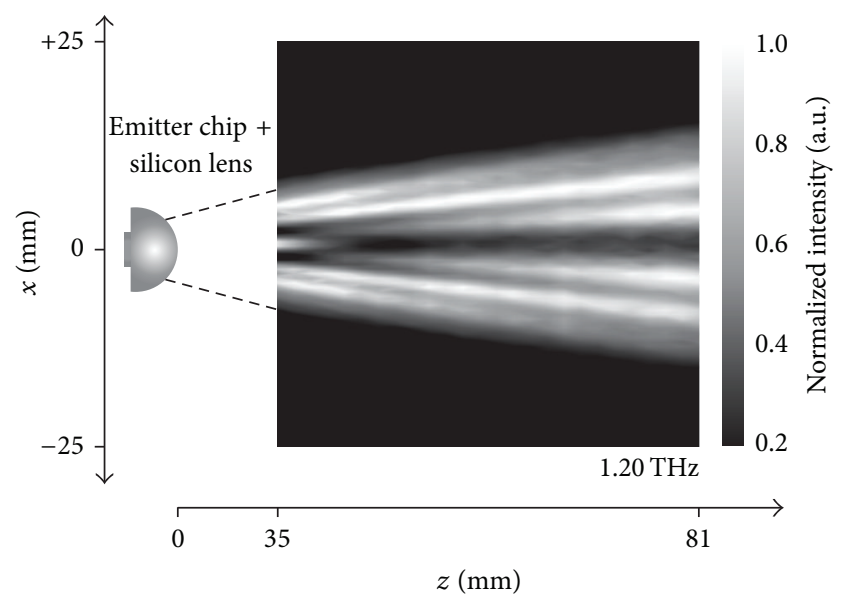

(b)

FIGURE 4: Schematic drawing of the setup for measuring radiation patterns (a). The fiber-coupled THz detector is movable in $x$-, $y$-, and $z$-direction with stepper motors. At each position a TDS scan can be performed. The dimension of the active area of the detector antenna is considerably small compared to the $\mathrm{THz}$ wavelength. An example of a measured beam profile in $X Z$-plane is shown for a better understanding of the results (b).

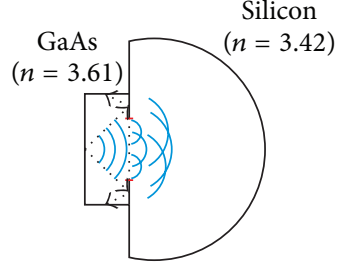

(a)

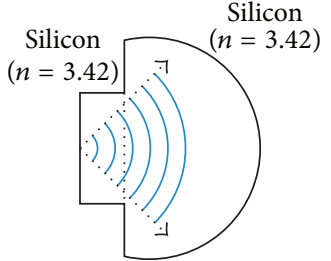

(b)

Figure 5: Schematic drawing of the THz wavefront propagation from GaAs substrate (a) and Si substrate (b) in a Si lens. In the case of a refractive index jump between the substrate and the lens a limiting stop aperture appears, leading to additional interference distortions of the $\mathrm{THz}$ radiation pattern. The stop aperture size in the case of Si substrate is determined by the lens size. The antenna sits in the center at the leftmost interface.

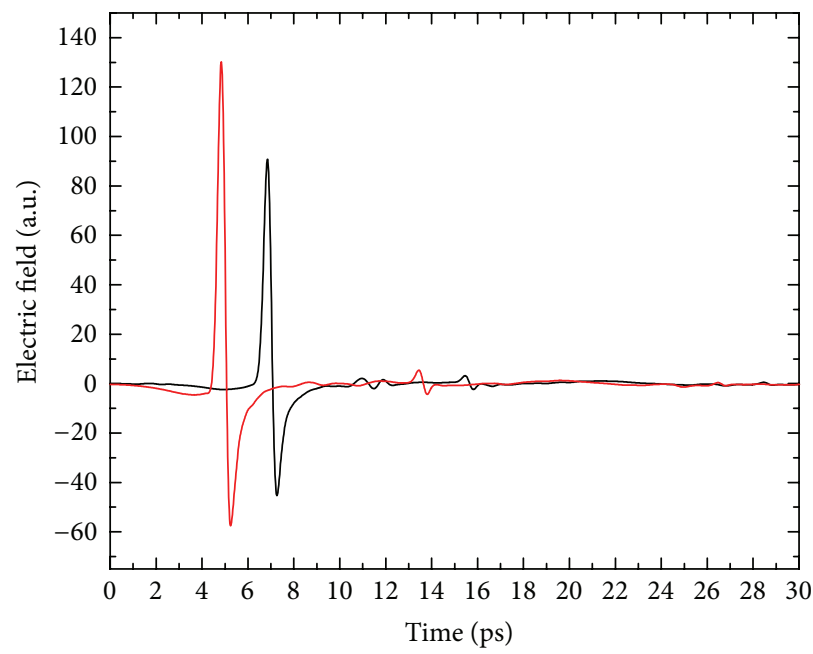

LT-GaAs on silicium substrate

— LT-GaAs on SI-GaAs substrate

(a)

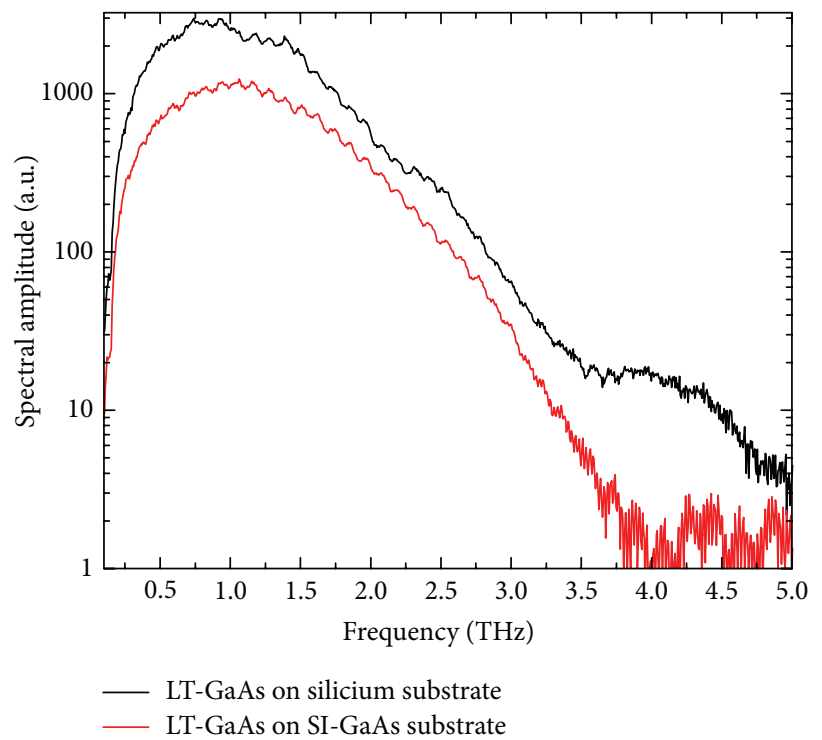

(b)

FIgURE 6: Measured waveforms (a) and corresponding spectrum (b) using Si or SI-GaAs substrate. 

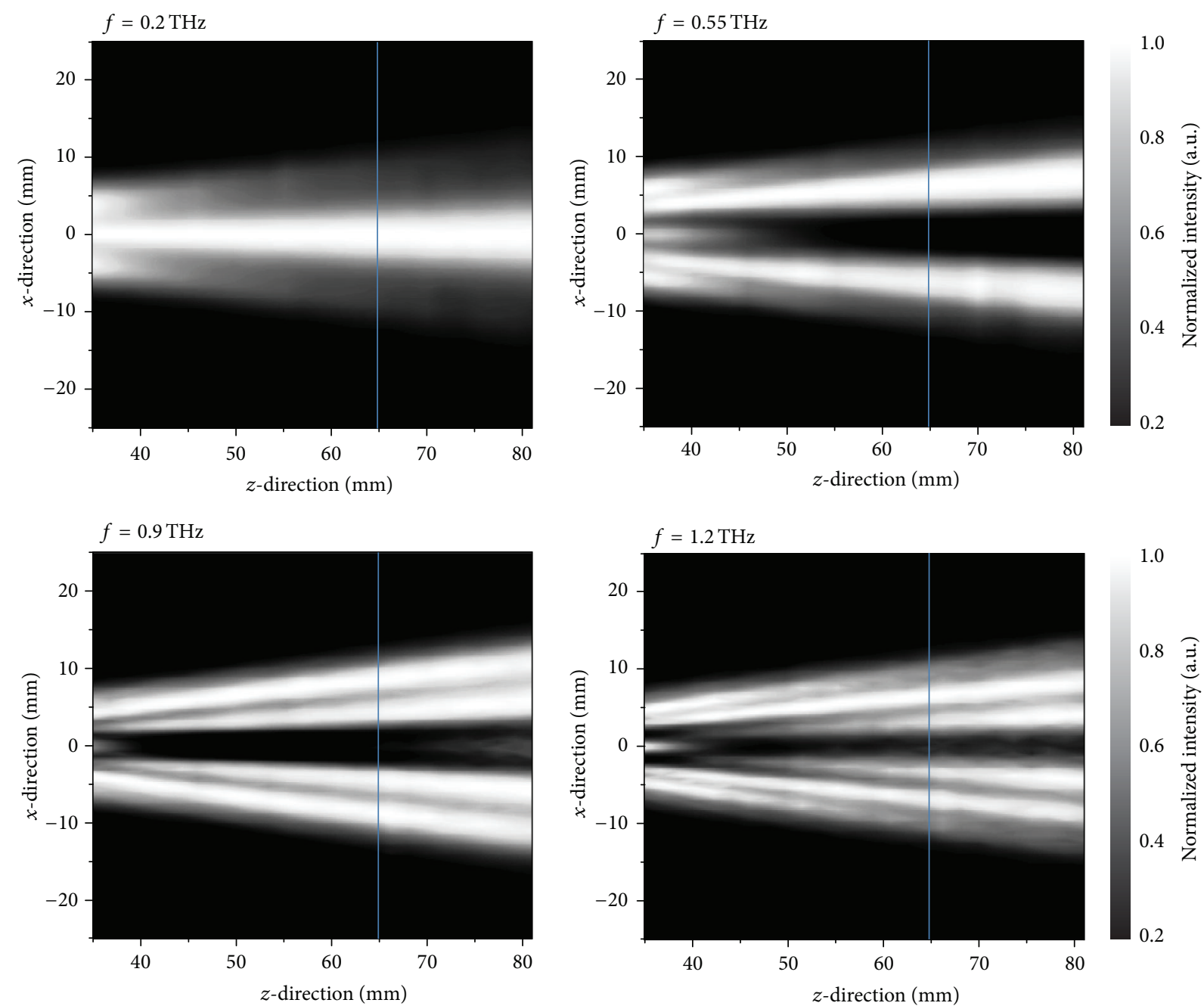

FiguRE 7: THz patterns at different distances to the detector in a range of 35 to $80 \mathrm{~mm}$ in $z$-direction and $-25 \mathrm{~mm}$ to $25 \mathrm{~mm}$ in $x$-direction for $0.2 \mathrm{THz}, 0.55 \mathrm{THz}, 0.9 \mathrm{THz}$, and $1.2 \mathrm{THz}$. The blue lines mark the position for the spectrally resolved distributions shown in Figure 8 .

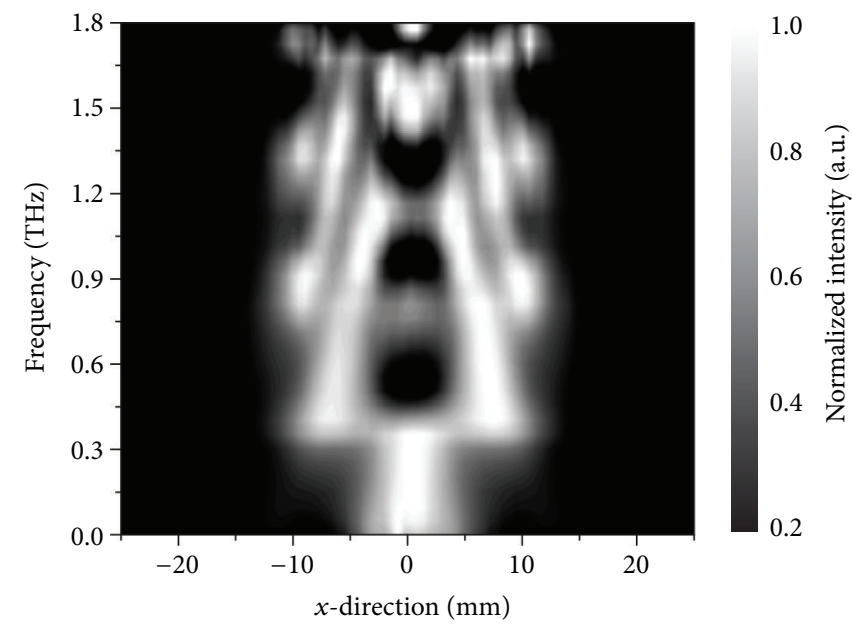

(a)

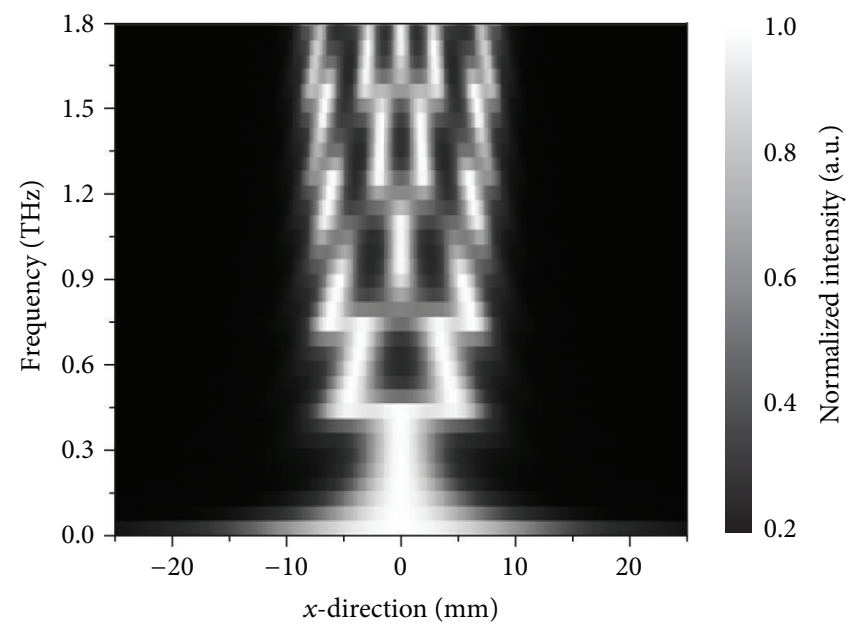

(b)

FIGURE 8: Measurement (a) and simulation (b) of the THz radiation pattern for LT-GaAs on SI-GaAs substrate as emitter at a distance of $65 \mathrm{~mm}$ in $z$-direction. Interference minima in both cases are clearly seen. 


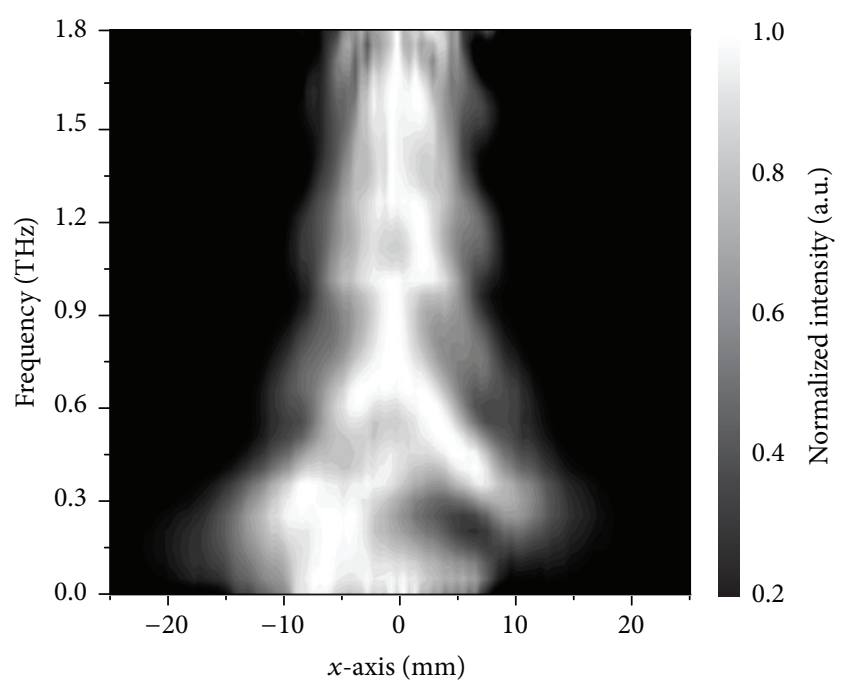

FIGURE 9: Measurement of the THz radiation pattern for LT-GaAs as emitter on HR-Si substrate with a spectral bandwidth up to $1.8 \mathrm{THz}$ at a distance of $65 \mathrm{~mm}$ in $z$-direction.

Besides the demonstrated advantage of using HR-Si as substrate material for the antennas, this combination is also promising from an economical point of view: The price of production for a single antenna can be essentially reduced, as only very small amounts of LT-GaAs are needed for successful antenna fabrication. Furthermore, this approach reduces the impact on the environment by reducing the overall amount of GaAs used.

Finally, we compare this also to an alternative source of $\mathrm{THz}$ emission, being simpler in application at first sight: so-called surface emitters based on moderately doped bulk semiconductors, for example, indium arsenide (see [17] and the references therein). Although the material in itself is much cheaper and does not require structuring, it needs much higher optical pump powers up to 1 watt or even higher to reach comparable average power in the $\mathrm{THz}$ beam. This makes the overall setup less flexible and suitable only for stationary laboratory systems. With compact and fiber-coupled antenna-based $\mathrm{THz}$ systems [18] requiring only severaltens-of-milliwatt optical powers combined with the improved spatial beam profile this seemingly old-fashioned approach of $\mathrm{THz}$ generation is still extremely attractive and fit for future applications.

\section{Conclusion}

We have presented radiation pattern measurements of dipole antennas fabricated using two different emitter substrates. For LT-GaAs on SI-GaAs as emitter substrate strong interference effects and higher-order diffraction effects are observed due to the refractive index step between the emitter substrate and the Si lens. These effects can be avoided by using LT-GaAs on HR-Si substrate resulting in almost interference-free beam propagation.

In this paper we have demonstrated the improved beam pattern by using HR-Si substrate instead of SI-GaAs substrate due to the near-perfect index matching between substrate and Si lens. We do not expect that the HR-Si substrate itself enhances the electric field of the $\mathrm{THz}$ output. The contact between the LT-GaAs layer and the HR-Si substrate is very critical to the $\mathrm{THz}$ output power. Any scratch or dust in between these two layers will cause a significant reduction of output power. Therefore, a simple comparison of the obtained $\mathrm{THz}$ output power is not sufficient to decide the influence of the substrate on the $\mathrm{THz}$ generation. Here, the process of the placement of the LT-GaAs layer on the HR-Si substrate has to be improved before this effect can be investigated.

The generation of almost interference-free $\mathrm{THz}$ beam will improve the quality of imaging setup, especially when using a multipixel approach. In this approach, the optical system can be described in a very simple way, for example, like a source with frequency dependent angle of radiation.

For our experiments, we use an antenna design which is widespread in the terahertz time domain spectroscopy. The goal of the paper is the proof of concept that the use of HRSi substrate instead of SI-GaAs will minimize interference pattern of the antenna output. Due to this radiation pattern, $\mathrm{THz}$ system can be improved by engineering the antenna and/or the imaging optics.

\section{Conflict of Interests}

The authors declare that there is no conflict of interests regarding the publication of this paper.

\section{Acknowledgment}

The authors thank Carsten Matheis for fabricating the antenna structures.

\section{References}

[1] S. L. Dexheimer, Ed., Terahertz Spectroscopy, CRS Press, 2008.

[2] G.-S. Park, Y. H. Kim, H. Han et al., Eds., Convergence of Terahertz Sciences in Biomedical Systems, Springer, 2012.

[3] J. F. Federici, B. Schulkin, F. Huang et al., “THz imaging and sensing for security applications-explosives, weapons and drugs," Semiconductor Science and Technology, vol. 20, no. 7, pp. S266-S280, 2005.

[4] H.-B. Liu, H. Zhong, N. Karpowicz, Y. Chen, and X.-C. Zhang, "Terahertz spectroscopy and imaging for defense and security applications," Proceedings of the IEEE, vol. 95, no. 8, pp. 15141527, 2007.

[5] F. Ospald, W. Zouaghi, R. Beigang et al., "Aeronautics composite material inspection with a terahertz time-domain spectroscopy system," Optical Engineering, vol. 53, no. 3, Article ID 031208, 2014.

[6] T. Yasui, T. Yasuda, K.-I. Sawanaka, and T. Araki, “Terahertz paintmeter for noncontact monitoring of thickness and drying progress in paint film," Applied Optics, vol. 44, no. 32, pp. 68496856, 2005.

[7] D. H. Auston, K. P. Cheung, and P. R. Smith, "Picosecond photoconducting Hertzian dipoles," Applied Physics Letters, vol. 45 , no. 3 , article 284, 1984. 
[8] A. P. DeFonzo and C. R. Lutz, "Optoelectronic transmission and reception of ultrashort electrical pulses," Applied Physics Letters, vol. 51, no. 4 , p. 212, 1987.

[9] C. Fattinger and D. Grischkowsky, "Point source terahertz optics," Applied Physics Letters, vol. 53, no. 16, p. 1460, 1988.

[10] M. van Exter, C. Fattinger, and D. Grischkowsky, "High brightness terahertz beams characterized with an ultrafast detector," Applied Physics Letters, vol. 55, p. 337, 1989.

[11] P. U. Jepsen, R. H. Jacobsen, and S. R. Keiding, "Generation and detection of terahertz pulses from biased semiconductor antennas," Journal of the Optical Society of America B, vol. 13, no. 11, pp. 2424-2436, 1996.

[12] M. Tani, S. Matsuura, K. Sakai, and S.-I. Nakashima, "Emission characteristics of photoconductive antennas based on lowtemperature-grown GaAs and semi-insulating GaAs," Applied Optics, vol. 36, no. 30, pp. 7853-7859, 1997.

[13] D. Grischkowsky, S. Keiding, M. van Exter, and C. Fattinger, "Far-infrared time-domain spectroscopy with terahertz beams of dielectrics and semiconductors," Journal of the Optical Society of America B, vol. 7, no. 10, pp. 2006-2015, 1990.

[14] D. Mittleman, Sensing with Terahertz Beams, Springer, 2003.

[15] J.-M. Rämer, F. Ospald, G. von Freymann, and R. Beigang, "Generation and detection of terahertz radiation up to $4.5 \mathrm{THz}$ by low-temperature grown GaAs photoconductive antennas excited at $1560 \mathrm{~nm}$," Applied Physics Letters, vol. 103, Article ID 021119, 2013.

[16] P. U. Jepsen and S. R. Keiding, "Radiation patterns from lenscoupled terahertz antennas," Optics Letters, vol. 20, no. 8, p. 807, 1995.

[17] J.-F. Lampin and E. P. Peytavit, "Integrated terahertz antenna and transmitter and/or receiver, and a method of fabricating them," French patent application FR0610164 filed on Nov. 21st 2006, International application PCT/FR2007/001912 filed on Nov. 21st 2007.

[18] F. Ellrich, T. Weinland, D. Molter, J. Jonuscheit, and R. Beigang, "Compact fiber-coupled terahertz spectroscopy system pumped at $800 \mathrm{~nm}$ wavelength," Review of Scientific Instruments, vol. 82, Article ID 053102, 2011. 

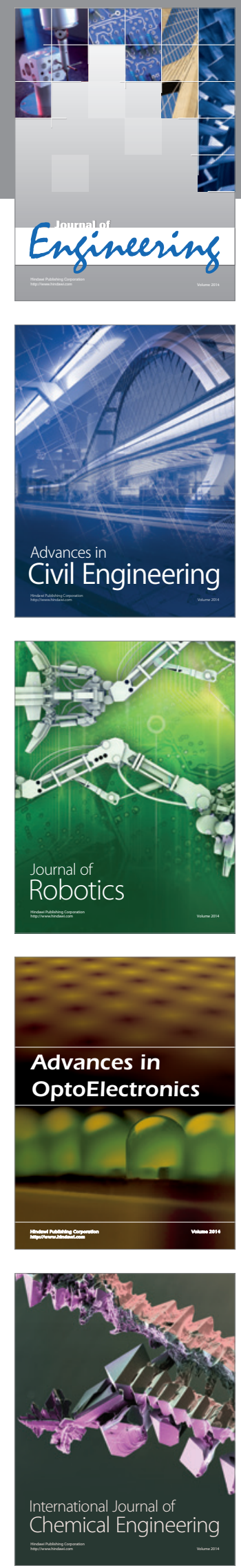

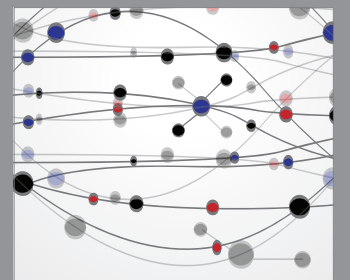

The Scientific World Journal
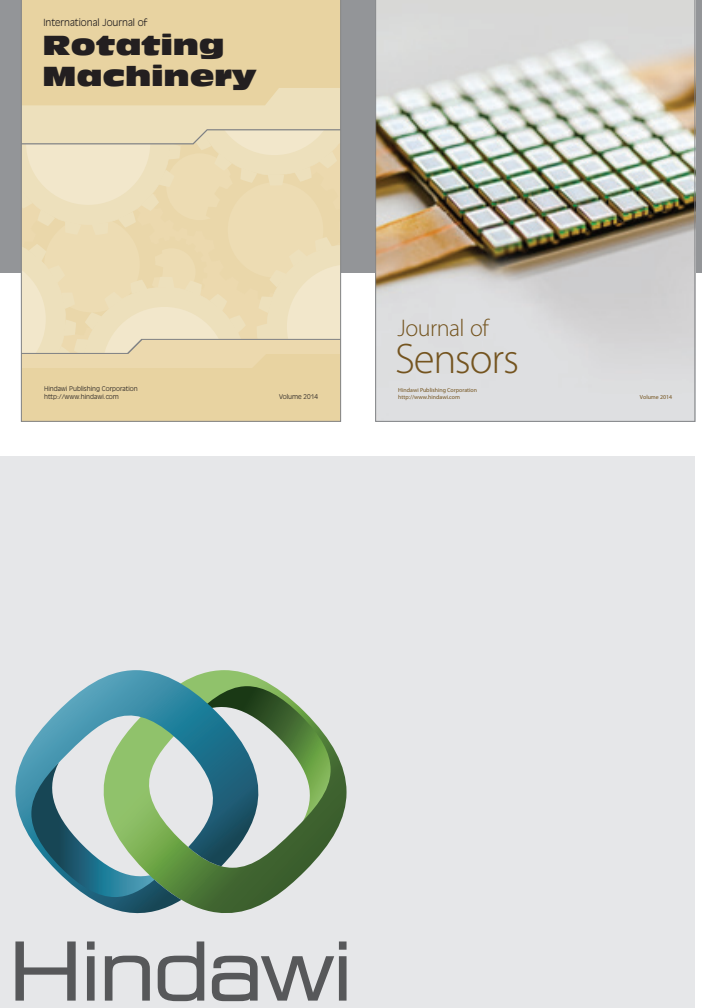

Submit your manuscripts at http://www.hindawi.com
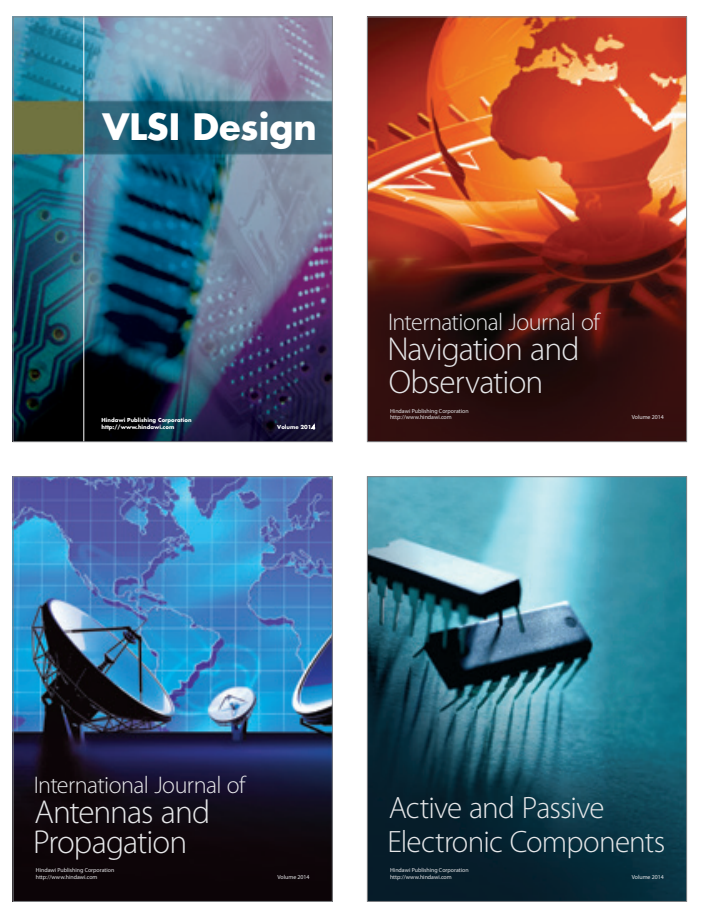
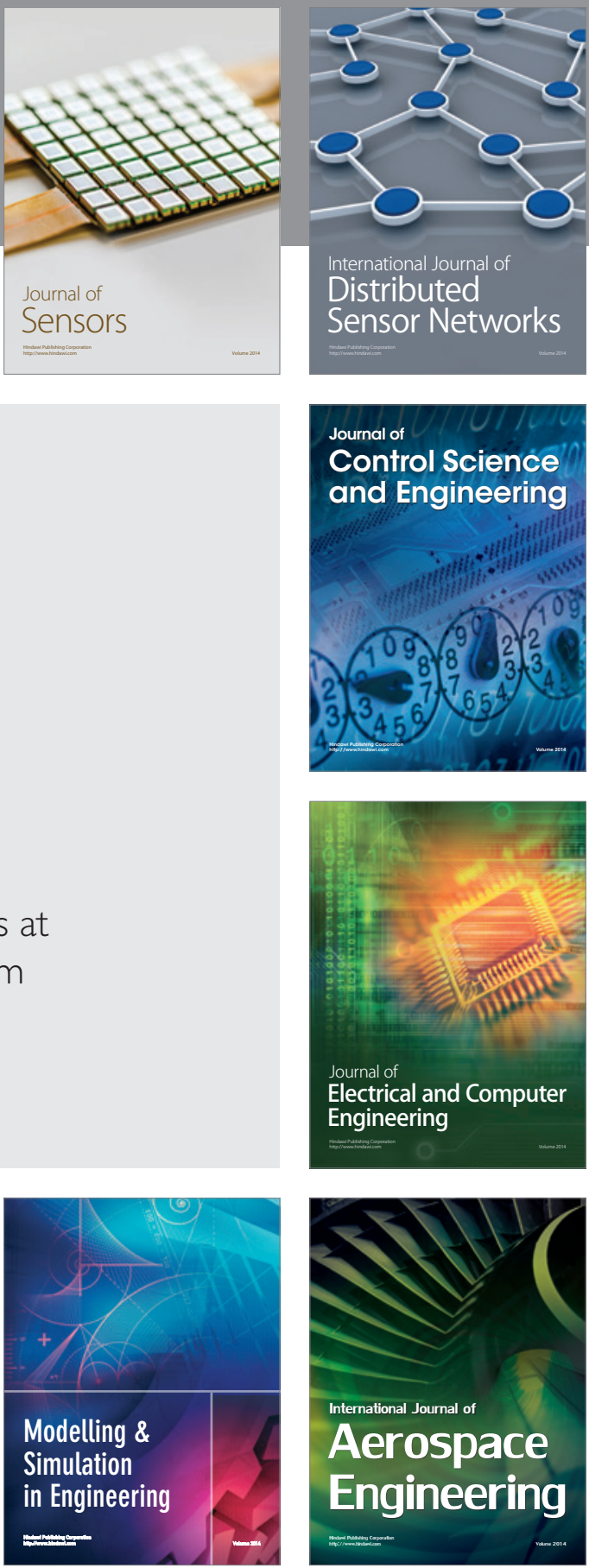

Journal of

Control Science

and Engineering
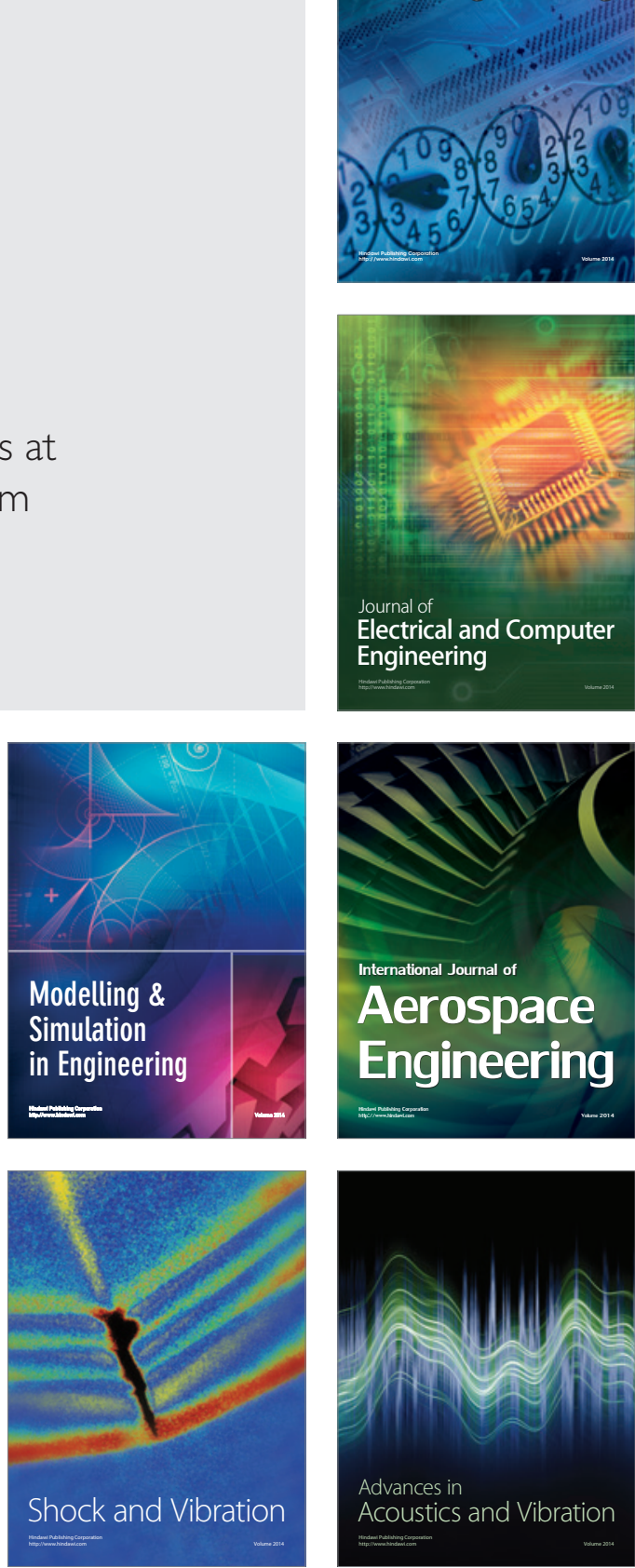